\title{
UNE SCIENCE DU LANGAGE POUR UNE SCIENCE DE L'HUMAIN
}

\section{(A language science for human sciences)}

\section{(Uma ciência da linguagem para as ciências humanas)}

\author{
Jean-Paul BRONCKART \\ (Faculté de Psychologie et des Sciences de l'Education \\ - Université de Genève)
}

Résumé: L'auteur de cet article propose d'abord une analyse de l'évolution des sciences du langage au cours des dernières décennies, qui fait apparaître la difficulté d'identifier un cadre épistémologique susceptible de se substituer au défunt programme chomskyen. Il soutient ensuite que l'enjeu central des sciences sociales est de comprendre comment s'est constitué, et comment se développe, l'espace gnoséologique proprement humain, et il examine à ce propos les apports des courants du cognitivisme, du constructivisme (de Piaget) et de l'interactionnisme social. L'auteur procède alors à une description détaillée de divers apports des sciences du langage: la conception saussurienne du statut des signes ainsi que les rapports entre textes et langues; la conception de Volochinov du rôle des discours dans le développement social; les approches de l'architecture des textes. Sur ces bases, il démontre enfin en quoi les sciences du langage peuvent fournir un appui décisif aux sciences de l'homme.

Mots-clé: Sciences humaines; Sciences du langage; Signe; Interactionnisme social; Texte.

Abstract: In this article, the author first of all presents an analysis of the evolution of language sciences during the past few decades, which points to the difficulty of identifying an epistemological framework which could constitute an alternative to the now extinct Chomskyan programme. Secondly, he claims that the major issue that the social sciences bave to deal with is to understand the emergence and development of specifically buman gnoseology; in this respect, he examines the contributions of cognitive science, of Piagetian constructivism and of social interactionism. The author then proceeds to a detailed description of several contributions of the language sciences: Saussure's conception of the status of signs and of the interactions between natural languages and texts; Volochinov's conception of the role of discourse in social development; different approaches of text architecture. Finally, on these grounds, the author demonstrates in what sense the language sciences can bring a decisive contribution to the buman sciences.

Key-words: Human sciences; Language sciences; Sign; Social interactionism; Text.

D.E.L.T.A., 27:2, $2011(337-361)$ 
Resumo: Neste artigo o autor começa por apresentar uma análise da evolução das ciências da linguagem durante as últimas décadas, que aponta para a dificuldade para identificar um arcabouço epistemológico que possa constituir uma alternativa ao agora extinto programa Chomskiano. Em segundo lugar, argumenta que o maior desafio a ser enfrentado pelas ciências humanas é entender a emergência e desenvolvimento de noseologia especificamente bumana; a esse respeito examina as contribuições da ciência cognitiva, do construtivismo Piagetiano e do sóciointeracionismo. A seguir o autor prossegue detalbando a descrição de várias contribuiçôes das ciências da linguagem: a concepção de Saussure do status do signo e da interação entre linguas naturais e textos; a concepção de Volochinov do papel do discurso no desenvolvimento social; diferentes abordagens da arquitetura do texto. Finalmente, com base nisso, o autor demonstra em que sentido as ciências da linguagem podem trazer uma contribuição definitiva às ciências humanas.

Palavras-chaves: Ciências Humanas, Ciências da linguagem, Signo, Interacionismo social, Texto.

Si elle a pu être considérée, lors de la période d'expansion du courant générativiste, comme la "discipline-pilote" des sciences humaines, la linguistique a depuis lors manifestement perdu ce statut, notamment en raison du doute qui s'est progressivement installé quant à la faisabilité et la crédibilité du programme chomskien. Le propos de cet article sera cependant de montrer que cette discipline demeure centrale, dans la mesure où les objets auxquels elle s'adresse constituent les éléments constitutifs des modalités spécifiquement humaines d'organisation psychique et sociale; ou encore de montrer que cette discipline peut fournir un appui décisif au développement d'une science intégrée de l'bumain, qui saisirait ce dernier, non dans sa seule dimension biologique, mais tout autant dans ses dimensions historique, sociale et culturelle.

Pour ce faire, nous procéderons d'abord à un bref examen des orientations épistémologiques prises par la linguistique aux cours des dernières décennies. Nous exposerons ensuite, depuis le cadre de l'interactionnisme social en lequel nous nous inscrivons, la problématique nodale d'une science intégrée de l'humain, qui a trait aux conditions d'élaboration et de développement d'un espace gnoséologique en permanence accessible et dynamique. Enfin, après avoir montré que certaines œuvres linguistiques, dont celles de Saussure et de Volochinov en particulier, ont d'ores et déjà fourni des analyses et propositions absolument décisives pour la clarification de cette problématique, nous nous risquerons à énoncer les conditions sous lesquelles la linguistique contemporaine pourrait contribuer à la nécessaire "reconfiguration" des sciences humaines. 


\section{QuelQues REMARQUeS SUR L'ÉVOLUTION RÉCENTE}

\section{DES SCIENCES DU LANGAGE}

Notre commentaire portera d'abord sur la Grammaire Générative et Transformationnelle (ci-après GGT), dont le projet épistémologique a été explicitement annoncé par son initiateur : la GGT avait pour but de fournir une représentation formelle explicite des "structures mentales innées » sous-tendant le langage humain et rendant ce faisant possible l'acquisition de toute langue naturelle. Pour Chomsky cette faculté de langage serait d'ordre strictement syntaxique: l'appareil mental humain comporterait d'un côté un composant relevant d'une sémantique universelle, c'est-à-dire un ensemble d'entités cognitives préconstruites et indépendantes du langagier, d'un autre côté un composant constitué des ressources phonétiques et signalétiques propres à l'espèce, et le langage proprement dit consisterait en un système de règles de couplage de ces deux ordres d'entités, système universel que langues naturelles matérialiseraient avec quelques variantes secondaires ou « superficielles »:

« [... $]$ il convient de se demander comment représenter les sons et les sens. Comme nous nous penchons sur les langues humaines en général, de tels systèmes de représentation devraient être indépendants de toute langue particulière. Nous devons, en d'autres termes, développer une phonétique et une sémantique universelles, qui délimiteront respectivement l'ensemble des signaux possibles et l'ensemble des représentations sémantiques possibles, pour toute langue humaine. On pourra alors parler d'une langue comme d'un couplage particulier de signaux avec des interprétations sémantiques, et étudier les règles qui établissent ce couplage. » (1969, p. 132)

Ce type de projet s'inscrit de fait dans une longue tradition qu'à la suite de Rastier (2001) nous qualifierons de logico-grammaticale qui, après Descartes et l'émergence du sujet pensant, a donné lieu aux quatre postulats suivants.

(a) Les processus de noèse (ou de pensée pure) sont autonomes et premiers par rapport aux processus de sémiose : le langage relève de ce fait d'un mécanisme secondaire, d'expression de structures cognitives qui en conditionnent inévitablement la structuration propre.

(b) Ces processus de noèse ont un fondement naturel; ils tiennent à l'équipement spirituel dont disposerait l'espèce humaine, équipement lui-même à base génétique ou inné. 
(c) En conséquence, les signes des langues ne peuvent que reformater approximativement des unités de pensée qui, elles, seraient stables en raison de leur fondement naturel ; signes qui, conformément à (a) ne jouent aucun rôle dans la constitution de ces unités de pensée.

(d) En conséquence encore, dès lors que les structures langagières sont censées refléter des opérations cognitives en droit universelles, il devrait exister un langage unique et idéal, ou à tout le moins une organisation structurelle commune à toutes les langues naturelles.

Les positions de ce type se révèlent de fait inaptes à traiter sérieusement de deux "réalités" pourtant indiscutables : l'extraordinaire variété des langues naturelles et les changements qui les affectent en permanence. S'agissant des causes et des déterminations de la variété lexicale et structurelle des langues, si aux XVIIe et XVIIIe avaient été invoquées les " passions » des peuples, qui déforment les structures linguistiques universelles, pour les générativistes ce problème est renvoyé à des différenciations d'ordre socioculturel, secondaires et indépendantes des mécanismes centraux de la pensée et des structures langagières universelles. Quant aux processus de changement, ils n'ont de fait dans ce cadre aucune explication possible: dans la mesure où le langage serait fondé sur des capacités cognitives biologiquement inscrites, pourquoi et comment ses manifestations pourraient-elles être sensibles au Temps et à l'Histoire? Ce qui explique la tonalité (au moins implicitement) fixiste de cette approche, les transformations caractérisant la vie des langues étant considérées comme des phénomènes ne relevant pas de linguistique proprement dite, ou encore comme tenant aux aléas des «performances » humaines.

Alors que Chomsky a de fait abandonné son projet épistémologique initial, pour d'autres raisons néanmoins que celles qui viennent d'être évoquées ${ }^{1}$, de multiples autres courants de linguistique ont entre temps émergé, qu'il serait inutile et prétentieux de vouloir décrire exhaustivement, mais à propos desquels nous formulerons les quelques remarques qui suivent.

1. Chomsky considère désormais que le langage humain se situe dans le prolongement direct des systèmes de communication animale, que la récursivité est sa seule propriété spécifique, et que l'émergence de cette propriété résulte de l'évolution de capacités autres, dont notamment les relations sociales! (cf. Hauser et al., 2002). 
A nous en tenir à la linguistique synchronique, la majorité des courants contemporains ont pris une orientation inverse à celle de la GGT et de la tradition logico-grammaticale, en saisissant résolument le langage dans sa dimension praxéologique (plutôt que structurale) et centrant en conséquence les analyses sur les rapports de co-dépendance entre les entités langagières et leur entour externe. Certains courants s'en tiennent à des entités linguistiques de l'ordre de l'énoncé, dont sont examinées les modalités d'interaction avec divers facteurs du contexte, soit dans ses dimensions essentiellement physiques comme dans la version princeps de la théorie de l'énonciation (cf. Benveniste, 1974), soit dans ses dimensions comportementales/sociales, comme dans la version initiale de la théorie des actes de langage, soit encore dans ces deux dimensions dans la plupart des développements contemporains d'orientation énonciative ou pragmatique. D'autres courants se centrent plutôt sur des entités de l'ordre du texte et/ou du discours, qui peuvent être analysées plutôt sous l'angle de leurs régularités de composition interne comme dans l'approche initiale d'Adam (1990), plutôt sous l'angle de leurs co-dépendance directe avec les structures comportementales et sociales d'échange, comme dans les diverses formes de linguistique interactionnelle, ou plutôt encore au titre de genres socialement formatés entretenant des rapports indirects avec les réseaux d'activité collective.

Cette floraison de recherches a sans nul doute fourni, en de multiples domaines, des éléments de description technique à la fois abondants et d'une réelle importance, mais elle est aussi le signe d'une sorte d'éparpillement théorique, qui se manifeste notamment dans la multitude des appellations d'école aujourd'hui observables dans la discipline. En outre, dès lors qu'ils se proposent d'aborder les entités langagières dans leurs rapports avec le contexte et/ou le co-texte, les courants qui viennent d'être évoqués sont immanquablement conduits à solliciter des références disciplinaires autres, ayant trait notamment aux caractéristiques des organisations sociales ou des interactions communicatives, ou aux opérations psychologiques mobilisées dans les décisions requises par l'activité langagière. Et à nous limiter à notre discipline d'origine, force est de constater que les emprunts notionnels à la psychologie présentent un caractère la plupart du temps conjoncturel: appui sur une conception de la subjectivité d'inspiration phénoménologique dans certains courants énonciatifs; sollicitation du cognitivisme modulaire d'inspiration fodorienne chez les tenants de la linguistique textuelle; mélange détonnant de behaviorisme et de cognitivisme dans certains 
courants pragmatiques, etc. Cet éparpillement des écoles et ce caractère ad hoc des emprunts aux autres sciences sociales/humaines témoignent à nos yeux d'un seul et même phénomène: la difficulté actuelle de la discipline d'articuler ses travaux empiriques à un projet épistémologique plus ample susceptible de se substituer au défunt programme chomskyen.

Formulé de la sorte, ce diagnostic est évidemment excessif, divers linguistes ayant bien évidemment proposé des directions de réorientation globale de la discipline. Rastier a notamment, dans de nombreux ouvrages (cf 1989; 2001), préconisé avec force une réorganisation des démarches linguistiques dans le paradigme global de l'herméneutique, et il proposé à cet effet un cadre conceptuel cohérent, portant à la fois sur les modalités d'interaction des genres de textes avec leur entour, et sur les niveaux d'organisation interne de ces mêmes textes. Si elle est d'un intérêt considérable et si nous nous en inspirons dans nos propres travaux actuels (cf. Bronckart, 2008), cette démarche nous paraît cependant présenter aussi un inconvénient, qui suscitera notre ultime remarque. Malencontreusement présentée par les rédacteurs du Cours de linguistique générale comme une entité fermée et statique, disjointe de l'activité de parole, « la langue » a depuis lors mauvaise réputation, et les excès du générativisme ont encore renforcé cette attitude de rejet. Mais au-delà des aléas de sa définition, cette notion s'adresse néanmoins à un évident domaine de réalité, qui est celui du vécu d'un idiome particulier et de sa systématique par les individus singuliers aussi bien que par les communautés. Et dès lors, s'il convient sans nul doute de rejeter la saisie logico-grammaticale de cette réalité, il n'y a pas lieu pour autant de nier l'existence et l'importance du phénomène « langue », comme semble le préconiser Rastier, à l'instar de certains tenants des approches contextualistes. Une science du langage ne peut "jouer" les textes/discours contre la langue, ou l'inverse; elle doit nécessairement penser l'articulation de ces deux dimensions, et c'est notamment à cette condition qu'elle pourra selon se restructurer en même temps qu'elle pourra contribuer à la réorientation des sciences humaines.

\section{LES ENJEUX D'UNE SCIENCE DE L'HUMAIN}

L'éparpillement des écoles se manifeste aussi dans les autres sciences humaines, mais d'une manière partiellement différente: en psychologie et en sociologie notamment se sont développés et se maintiennent depuis un 
siècle de grands cadres épistémologiques; mais ceux-ci sont profondément divergents, et cette divergence tient essentiellement à la position adoptée à l'égard d'une seule et même problématique nodale: comment s'est élaboré et comment se développe l'espace gnoséologique humain, et partant quel est le statut qui peut lui être accordé ?

Par « espace gnoséologique », nous entendons les multiples formes de connaissances qui se sont élaborées au cours de l'histoire sociale et qui se transmettent de générations en générations. Cet espace est donc constitué d'entité idéelles ou psychiques signifiantes, mais celles-ci ont en fait deux lieux d'ancrage distincts: dans l'intériorité ou la pensée consciente des individus d'une part, dans des construits sociaux d'autre part que Dilthey (1883/1992) avait qualifiés de «mondes d'œuvres et de culture » et dont Habermas a analysé les modalités d'organisation en termes de "mondes formels de connaissances » (1987). Dans Représentations individuelles et représentations collectives (1898), Durkheim avait proposé une analyse particulièrement puissante de ces deux ancrages, en montrant que, tout comme les entités psychiques individuelles ont un statut et des modalités d'organisation clairement distincts de ceux du substrat neurophysiologique auxquelles elles s'adossent matériellement, les entités psychiques collectives témoignent d'une réelle autonomie statutaire et organisationnelle eu égard aux institutions et aux autres formes d'organisation sociale qui en sont le support. Et cette autonomie de l'idéel ne peut pas être sans rapport avec l'émergence du langage, qui constitue un véhicule important de tout type de représentation, et qui est également la condition sine qua non de la transmission de ces représentations au cours de l'histoire. La question de fond de toute science de l'humain est donc de savoir comment se sont constituées des connaissances ayant comme spécificité d'être sémiotiques et signifiantes, de s'ancrer dans des organismes qui en deviennent des personnes dotées d'une pensée conscience, et de s'ancrer également dans les formes d'organisation et les productions culturelles des communautés.

Nous défendrons une manière d'aborder cette question reposant sur des orientations qui, pour être fermes, sont néanmoins en soi discutables. Il s'agit d'abord de se placer dans la perspective de l'évolution des espèces, ce qui conduit à prendre en compte l'existence, au niveau du vivant et plus particulièrement des animaux "supérieurs", de capacités psychiques individuelles ainsi que de modalités d'organisation des activités collectives. La question qui nous occupe doit donc être posée en termes de continuité et 
de rupture; il convient de montrer en quoi les modes de fonctionnement humain s'ancrent sur (et prolongent) les modes de fonctionnement du monde animal, en même temps qu'il convient de montrer comment, sur cette base, ont émergé les spécificités évoquées plus haut. Mais il s'agit aussi de se placer dans une perspective plus large, inspirée du monisme matérialiste de Spinoza, dont nous ne pourrons évoquer ici que deux principes majeurs. Le principe du parallélisme psycho-physique tout d'abord, qui se décline en trois thèses. (a) Chaque forme issue de l'évolution de la matière, y inclus les substances inertes, comporte d'un côté des dimensions physiques ou observables (inscrites dans l'espace), d'un autre des dimensions processuelles ou psychiques non directement observables. Ces deux dimensions ne sont que deux faces d'une même réalité, et elles ne nous paraissent disjointes qu'en raison de la limitation de nos capacités cognitives (au plan ontologique donc, le psychique est tout aussi "réel" que le physique). (b) Les propriétés physiques observables et les propriétés des processus sous-jacents sont, dès lors, en chaque forme naturelle, nécessairement d'un niveau de complexité équivalent, en tout cas à l'état d'équilibre de ces formes. (c) Cependant, si les formes matérielles évoluent en permanence, c'est parce que les processus sous-jacents sont aussi susceptibles de créer, sous certaines conditions, des déséquilibres, qui entraînent eux-mêmes des rééquilibrations donnant naissance à des formes nouvelles; thèse qui est en fait au cœur des approches thermodynamiques contemporaines. Le second principe est celui du rôle central des interactions dans la constitution des entités psychiques de quelque forme naturelle que ce soit. Préfigurant la dialectique première de Hegel comme la conception du développement formulée Piaget, Spinoza soutient en effet que la connaissance procède des contacts entre corps/esprit d'une part, entités mondaines d'autre part, contacts qui entraînent d'abord la différenciation soi-monde, puis, en se répétant, la différenciation progressive des objets du monde.

L'adoption de ces principes conduit à récuser toutes les formes de dualisme issues de la bi-partition posée par Descartes entre ordre de l'âmepensée et ordre des corps et des objets; position qui revient à considérer que les dimensions psychiques processuelles seraient propres à l'humain, et qui ne constitue ce faisant qu'un reliquat du créationnisme religieux. Elle conduit aussi à rejeter les thèses des neurosciences et de certains courant cognitivistes radicaux, en ce que leur conception de l'évolution accorde un rôle exclusif à l'équipement biologique sans prendre en compte réellement cet autre facteur majeur que constitue l'évolution des modalités 
comportementales d'interaction entre les organismes et leur milieu; nous soutiendrons pour notre part qu'il n'y a pas détermination unilatérale du biologique sur le mental et le comportemental mais co-construction et co-détermination de ces trois registres. Il s'agit donc d'aborder la problématique des conditions de constitution de la gnoséologie humaine dans une perspective à la fois moniste, évolutionniste et "interactive", mais deux grands paradigmes adossés à ces principes restent aujourd'hui en concurrence, que nous qualifions respectivement d'interactionnisme logique et d'interactionnisme social.

L'orientation interactionniste logique est particulièrement illustrée par l'œuvre de Piaget, que l'on peut lire comme une tentative de validation, expérimentale et "génétique" (au sens de développementale), de la synthèse qu'avait proposé Kant (1781/1944) entre positions empiristes et rationalistes. Pour ce philosophe, d'un côté toute connaissance présuppose l'expérience, c'est-à-dire la mise en ouvre d'interactions entre le sujet et le milieu, au terme desquelles certaines des empiries sont enregistrées et conservées; mais d'un autre côté l'esprit analyse et organise ce matériau en lui appliquant des catégories rationnelles (Temps, Espace, Causalité, etc.) qui relèvent de ses propriétés intrinsèques ou a priori, cette application des catégories de la raison aux données expérientielles aboutissant aux diverses formes de jugement et de raisonnement humains. Piaget a adopté une position analogue, mais en contestant que les a priori soient innés et structuraux, et en tentant dès lors de montrer comment les opérations cognitives se construisent au cours de l'ontogenèse sous l'effet de processus interactifs généraux qui, eux, seraient innés ou hérités. Dans ses études sur la phase initiale du développement (de la naissance à 18 mois), il a montré comment le contact actif de l'organisme humain avec son milieu, initialement sous le contrôle des réflexes innés, génère progressivement, par le jeu des mécanismes d'assimilation et d'accommodation, des traces internes relatives aux propriétés des objets externes, ainsi qu'aux propriétés des comportements propres. Il a montré ensuite que, sous l'effet des mêmes mécanismes, ces traces s'organisent en ces configurations plus stables que sont les images mentales; images qui restent cependant dépendantes des objets et des comportements qui les ont suscitées, qui restent inaccessibles au contrôle conscient du sujet, et qui ne permettent ainsi qu'une intelligence sensori-motrice. Piaget a analysé ensuite les conditions sous lesquelles ce psychisme pratique se transforme en pensée consciente, en soutenant que ces sont les mêmes processus naturels d'interaction entre organisme et milieu 
qui provoquent la rencontre avec les entités figuratives disponibles dans le milieu (images, symboles, signes) et que l'intériorisation de ces éléments figuratifs rend possible la transposition des schèmes pratiques au niveau mental, c'est-à-dire l'émergence d'une intelligence opératoire et autoaccessible.

Comme nous l'avons montré (cf. Bronckart, 1997a), dans l'analyse de ses données, Piaget ne prend cependant en compte que les interactions entre un enfant singulier et le milieu en ce qu'il est physique; il néglige en d'autres termes le rôle que peuvent jouer les interventions formatives des adultes et les productions langagières nécessairement mobilisées dans ce cadre, considérant que ces éléments ne constituent pas de véritables facteurs de développement:

«Les opérations de la pensée [...] tiennent aux coordinations générales de l'action [...] et non pas au langage et aux transmissions sociales particulières, ces coordinations générales de l'action se fondant elles-mêmes sur les coordinations nerveuses et organiques qui ne dépendent pas de la société. » (1970, p. 177)

Et cette position l'a conduit à une sorte d'impasse qu'il a lui-même commentée dans un de ses derniers écrits $(1989)^{2}$. L'auteur y constate d'abord que les mécanismes physiologiques humains, à l'instar de tous les phénomènes physiques, fonctionnent selon une logique causale.

Il ajoute ensuite que ce fonctionnement causal s'applique aussi aux schèmes du sensori-moteur mais qu'il ne peut caractériser les mécanismes à l'œuvre dans la pensée consciente, dans la mesure où ces derniers relèvent d'une logique d'implication: ils consistent en enchaînements d'implications signifiantes, régis par des règles non nécessaires, ou d'ordre normatif:

«Sur le terrain de l'intelligence, le mode essentiel de liaison propre à la conscience logique est l'implication (...) selon laquelle une ou plusieurs affirmations en entraînent nécessairement une autre. Par exemple, la vérité de $2+2=4$ n'est pas «cause» de la vérité de $4-2=2$ (...) la vérité (...) de $2+2=4$ «implique» celle de $4-2$ $=2$, ce qui est tout autre chose. En effet, cette implication se caractérise par un sentiment de nécessité qui est bien différent d'une détermination causale, car celle-ci ne souffre pas d'exception, tandis que la nécessité constitue une obligation que l'on doit respecter : or ce n'est pas toujours ce que l'on fait, si bien que le logicien Lalande

2. Il s'agit de la version remaniée de L'explication en psychologie et le parallélisme psycho-physiologique, texte rédigé en 1974 et que nous citons dans la réédition de 1989. 
énonçait l'implication en disant « $p$ implique $q$ pour l'honnête homme» de manière à souligner son caractère normatif. » (1989, p. 177)

Piaget se pose alors la question de savoir comment un système causal peut, au cours du développement, se transposer directement en un système d'implications signifiantes, et finit par avouer qu'au plan empirique, ce problème reste pour lui sans véritable solution. Nous reviendrons plus loin sur les leçons à tirer de cette analyse, qui nous paraît capitale.

Le mouvement interactionniste social s'est développé dès les débuts du XXe, au travers des œuvres de Bühler, Dewey, Mead, Vygotski, Wallon et bien d'autres. Adossé au spinozisme et au marxisme, ce courant se caractérise, au contraire de Piaget, par une approche du développement humain qui prend résolument en compte les préconstruits de l'histoire sociale (les formes d'activité collective, les œuvres, les organisations sociales) ainsi que les processus de médiation formative, et qui accorde un rôle décisif aux pratiques sémiotiques et/ou langagières.

Figure de proue de ce mouvement, Vygotski (1934/1977) a proposé un schéma développemental que l'on peut résumer comme suit. (a) L'espèce humaine est biologiquement dotée de capacités nouvelles qui permettent le déploiement d'activités collectives plus complexes que celles des autres animaux socialisés. (b) La complexité de ces activités collectives a rendu nécessaire l'émergence d'un mécanisme d'entente dans l'agir, en l'occurrence l'émergence de l'activité langagière en tant qu'instrument de planification, de régulation et d'évaluation des autres formes d'activités. (c) Cette activité langagière est productrice d'unités sémiotiques, c'està-dire de représentations d'aspects du monde qui ne sont plus, comme dans le règne animal, inéluctablement idiosyncrasiques, mais qui, de par les conditions mêmes de leur constitution, sont partagées ou collectives. (d) C'est alors l'appropriation et l'intériorisation de ces unités sémiotiques socialisées qui engendrent la transformation du psychisme hérité en un système de pensée auto-accessible ou potentiellement conscient, et c'est le déploiement de l'activité langagière par des individus pensants qui rend possible la constitution des mondes d'œuvres et de culture. Selon cette approche, les images mentales des animaux (et des jeunes enfants) restent idiosyncrasiques parce qu'elles ne bénéficient d'aucun moyen propre de discrétisation et de stabilisation ; elles constituent une sorte de nébuleuse, pour laquelle la description que proposait Saussure de ce que pourrait être 
une " pensée sans langage » paraît (paradoxalement) éclairante: "prise en elle-même, la masse purement conceptuelle de nos idées, la masse dégagée de la langue représente une espèce de nébuleuse informe où l'on ne saurait rien distinguer dès l'origine. » (in Constantin, p. 285). L'intériorisation des signes du langage, non seulement rendrait possible cette discrétisation et cette stabilisation, entraînant une complète restructuration de l'appareil psychique, mais en outre, dès lors que ces signes sont pétris de significations sociohistoriques, elle entraînerait un changement de statut de cet appareil, qui passerait, selon la célèbre formule de Vygotski, de l'ordre du biologique à l'ordre de l'historico-culturel.

Nous adhérons pour notre part sans réserve à ce schéma, dont Volochinov a d'ailleurs proposé une version quasi identique (1929/1977 — cf. infra), mais il faut reconnaître que ces deux auteurs, s'ils mettaient l'accent respectivement sur le rôle des signes et des textes, n'ont proposé que des ébauches d'analyse du statut de ces derniers, et dès lors n'ont pas proposé de démonstration technique précise des mécanismes par lesquels les propriétés des entités langagières entraînent la restructuration du psychisme humain. Mais comme nous le verrons ci-dessous, une telle démonstration a de fait été proposée dans l'œuvre réelle de Saussure.

\section{QuelQues APPORTS DÉCISIFS DES SCIENCES DU LANGAGE}

Dans ce qui suit, nous solliciterons, parmi les multiples avancées des sciences du langage, quatre approches qui nous paraissent fournir un appui majeur aux thèses de l'interactionnisme social, les deux premières issues de l'œuvre de Saussure, la troisième de celle de Volochinov et la quatrième des courants actuels centrés sur l'analyse de l'organisation interne des textes.

\subsection{Des rapports entre langue et discours dans l'œuvre réelle de Saussure}

Si le terme de "discours » n'apparait pas dans le CLG, on en trouve néanmoins de multiples occurrences dans les notes manuscrites de Saussure ainsi que dans les cahiers d'étudiants ayant suivi ses cours. Sa position était en fait que les discours/textes constituent le milieu de vie premier des phénomènes langagiers: c'est dans le cadre de leur mise en œuvre synchronique, ainsi que dans le cours de leur transmission historique, que 
les valeurs signifiantes des signes se construisent, et qu'elles se transforment en permanence:

«Toutes les modifications, soit phonétiques, soit grammaticales (analogiques) se font exclusivement dans le discursif. Il n'y a aucun moment où le sujet soumette à une révision le trésor mental de la langue qu'il a en lui, et crée à tête reposée des formes nouvelles [...] qu'il se propose, (promet) de "placer" dans son prochain discours. Toute innovation arrive par improvisation, en parlant, et pénètre de là soit dans le trésor intime de l'auditeur ou celui de l'orateur, mais se produit donc à propos du langage discursif. » (2002, p. 95)

Qu'est-ce alors que LA LANGUE en regard de ces discours? Saussure en a donné une première définition lors des trois Conférences prononcées en 1891 lors de l'inauguration de sa chaire genevoise. Il y souligne d'abord l'inéluctable continuité des faits de langage et en vient à déclarer qu'il n'existe en fait qu'une seule langue, à l'œuvre depuis l'émergence de l'espèce: " j'insisterais encore une fois sur l'impossibilité radicale, non seulement de toute rupture, mais de tout soubresaut, dans la tradition continue de la langue depuis le premier jour même où une société humaine a parlé » (ibid., p. 163). Il pose ainsi que la langue constitue une entité ontologiquement une, ou encore qu'existe un stock de ressources qui seraient perpétuellement redistribuées dans le temps et dans l'espace, les diverses communautés situées dans ces coordonnées n'en exploitant, par convention, que des sous-ensembles restreints.

Mais dès ces mêmes Conférences, Saussure souligne aussi que cette langue universelle connaît des « états » successifs, et comme on le sait, c'est sur le statut de ces états qu'ont surtout porté ses réflexions et ses enseignements ultérieurs. Dans une première acception, la langue comme état, c'est un " réservoir» (ou un «trésor ») de valeurs signifiantes issues des textes, telles que celles-ci se « déposent » dans le « cerveau » du sujet parlant.

«Tout ce qui est amené sur les lèvres par les besoins du discours, et par une opération particulière, c'est la parole. Tout ce qui est contenu dans le cerveau de l'individu, le dépôt des formes entendues et pratiquées et de leur sens, c'est la langue. » (in Komatsu \& Wolf, Cours I, 1996, pp. 65-66)

Si Saussure considère ici que ce dépôt est localisé « dans le cerveau ", dans d'autres passages il peut mentionner tout autant « la conscience des sujets parlants » ou la «sphère associative interne », ces diverses expressions 
désignant manifestement ce que nous qualifions d'appareil psychique des personnes. Il ajoute que les formes intériorisées sont réorganisées dans cet appareil: elles y font l'objet de classements donnant lieu à la constitution de séries de termes entretenant entre eux des rapports de ressemblancedifférence, selon des critères d'ordre sonore ou sémantique. Si elles sont bien issues des textes, les entités signifiantes s'organisent donc dans la sphère associative interne sous des modalités différentes de celles de l'organisation linéaire de la textualité, et cette organisation constitue un premier "degré" d'état de langue, que nous qualifierons de langue interne. Mais Saussure a soutenu que la langue avait aussi son siège dans la collectivité: « La langue est l'ensemble des formes concordantes que prend [le] phénomène [de langage] chez une collectivité d'individus et à une époque déterminée » (2002, p. 129). Dans cette autre approche, il souligne que la langue demeure toujours sous le contrôle ultime du social, en l'occurrence des accords ou conventions qui s'y établissent. Cet état de langue collectif est donc le niveau où s'exerce l'activité normative des générations de locuteurs, et nous le qualifierons dès lors de langue normée ("degré" de langue qui est par ailleurs celui que tentent d'appréhender et de décrire les grammairiens ou linguistes).

Pour résumer la position de Saussure quant aux unités d'analyse possibles d'une science du langage, on peut considérer que celui-ci pose d'abord deux entités dont l'étude semble ne pas pouvoir relever de la seule linguistique : l'activité langagière d'une part, la langue universelle d'autre part, en tant que stock de ressources dont l'extension semble finie. Mais il soutient aussi que la mise en œuvre effective de ces deux entités par les groupes humains, dans des circonstances historiques et géographiques variables, requiert la prise en compte des trois autres entités, qui constituent les véritables objets d'une science du langage.

(a) Les textes/discours, comme premier lieu de vie des signes, dans le cadre desquels les valeurs de ces signes se re-fabriquent en permanence.

(b) La langue interne, comme système d'organisation psychologique des valeurs signifiantes extraites des textes, système qui est soumis aux contraintes conventionnelles de la langue normée dont attestent les textes, mais qui est marqué aussi par l'histoire de vie et les propriétés particulières des personnes; cette langue interne constituant un deuxième lieu de vie des signes. 
(c) La langue normée, comme système d'organisation des valeurs signifiantes des signes géré cette fois par les groupes sociaux et soumis à leurs normes propres de fonctionnement. Il s'agit là d'un troisième lieu de vie des signes, à caractère second ou abstrait: la "langue française", par exemple, n'existe qu'en tant que produit d'un travail de généralisation et d'abstraction, travail aux résultats variables et incertains comme chacun le sait.

Une telle approche rompt radicalement, on le constate, avec l'un des principes de la tradition logico-grammaticale et du générativisme, en ce qu'elle pose que, plutôt que d'être des produits de la mise en œuvre d'un hypothétique appareil langagier universel, les textes/discours sont au contraire à la source même de la constitution de la langue interne et de la langue normée. Elle conduit aussi à ne pas sous-estimer le rôle de la langue, dans la mesure où les trois entités identifiées sont fondamentalement interdépendantes et sont le siège d'un mouvement dialectique permanent: les signes et leurs valeurs sont mis en ouvre dans les textes; ils font l'objet d'une appropriation par les personnes et se réorganisent dans leur appareil psychique selon les modalités singulières (langue interne); ils sont ensuite extraits de ce même appareil pour être réinjectés dans de nouveaux textes, sous le contrôle de la langue normative, la dimension individuelle de ce dernier processus étant à l'origine des dimensions créatives des nouvelles productions, la dimension sociale de leurs dimensions reproductives.

\subsection{Du statut des signes et des conditions de leur constitution}

Dans l'Essence double, dans diverses notes et dans le Cours I, Saussure a décrit de manière détaillée les opérations impliquées dans la construction des signes; opérations qu'il a conçues comme se déployant simultanément, ou encore comme étant interdépendantes, comme en atteste sa désormais célèbre formulation du quaternion:

\footnotetext{
"Nous sommes toujours ramené aux quatre termes irréductibles et aux trois rapports irréductibles entre eux ne formant qu'un seul tout pour l'esprit: (un signe / sa signification $)=($ un signe / et un autre signe) et de plus = (une signification / une autre signification). [...] C'est là ce que nous appelons le QUATERNION FINAL et, en considérant les quatre termes dans leurs rapports: le triple rapport irréductible. » (2002, p. 39)
} 
Un premier mécanisme réside en la constitution des images acoustiques sur un versant, des images de sens sur un autre, par traitement des entités matérielles sonores ou des entités mondaines référentielles, mécanisme qui ne mobilise en réalité que les processus élémentaires d'assimilation, d'accommodation et d'équilibration dont Piaget a démontré qu'ils interviennent dans la constitution de tout type d'image mentale. Un second mécanisme réside en la sélection d'une image de chacun des deux registres, au sein d'un ensemble associatif à la fois socialement pertinent et personnel; le processus de différenciation-opposition ici impliqué est également élémentaire et mobilisable dans bien d'autres traitements cognitifs. Le troisième mécanisme consiste enfin en l'« accouplement » des deux images par association; association qui est certes constitutive des termes auxquels elle s'applique mais ce type d'association formative ne constitue pas un phénomène unique ou spécifiquement langagier; les behavioristes aussi bien que Piaget en ont décrit de nombreux exemples dans bien d'autres types de traitement cognitif.

Les processus mis en œuvre dans la construction des signes sont comme on le constate les processus interactifs hérités de l'évolution, c'est en cela que la démonstration saussurienne nous paraît capitale. Cette construction se situe donc dans le prolongement direct des processus communs au vivant : c'est l'aspect de continuité évoqué plus haut; mais une fois constitué, ces signes transforment radicalement le psychisme hérité et le font passer, comme disait Vygotski, du régime bio-comportemental au régime sociohistorique; c'est l'aspect de rupture également évoqué. Le signe est donc le lieu même de la continuité-rupture, et l'élément déterminant de la rupture humaine tient au fait que les processus hérités s'appliquent non plus seulement à des objets physiques comme dans le monde animal, mais à des objets sociaux, à ces " petits bruits émis par la bouche ", selon l'expression de Bloomfield, qui sont conventionnellement associés à des dimensions de l'activité humaine. En d'autres termes, les signes ont cette propriété radicalement nouvelle dans l'évolution de constituer des cristallisations psychiques d'unités d'échange social, et c'est cette socialisation du psychisme qui est fondatrice de l'humain. 


\subsection{Les discours et leur rôle dans le développement social et psychologique}

Volochinov $(1929 / 2010)^{3}$ a jeté les bases d'une approche radicalement nouvelle du statut des textes et de leur rôle dans le déploiement de la vie psychique et sociale, dont nous ne pourrons relever que trois aspects centraux dans le cadre de cette contribution.

Comme nombre de linguistes russes des années 20, cet auteur était engagé dans le débat sur le statut des œuvres littéraires/poétiques, et critiquait fermement la position des "formalistes", en soutenant que ces œuvres constituaient des produits de modalités particulières de communication sociale, et qu'elles devaient en conséquence être appréhendées en tant que formes textuelles spécifiques, en une démarche comparant leurs conditions de production et leurs propriétés internes avec celles des textes "ordinaires". Et c'est cette position continuiste qui l'a conduit à généraliser la notion de genre à toutes les sortes de textes, et à tenter de définir ces genres en se fondant essentiellement sur le type d'interaction sociale/verbale dont ils étaient le produit. Il a soutenu en outre que le méthodologie d'analyse des textes devait s'organiser en un programme descendant, consistant à étudier d'abord les activités d'interaction verbale dans leur cadre social, à identifier ensuite les genres textuels mobilisés dans ces interactions, et à procéder enfin à l'examen des multiples propriétés linguistiques formelles de chacun des genres. Programme qui a orienté, comme on le sait, une large part des courants contemporains d'analyse de discours.

Cette approche des textes s'inscrivait cependant pour Volochinov dans une perspective bien plus large, ayant trait au rôle que jouent les productions verbales dans le développement psychologique et social. Il soutenait d'un côté que les configurations de signes attestables dans les textes (ou, en termes saussuriens, la réélaboration permanente des valeurs de ces signes dans la textualité) constituaient une forme de réfraction de l'idéologie ambiante ou de la "psychologie du corps social », et d'un autre côté, adhérant explicitement aux thèses de Vygotski, il soutenait que c'est l'appropriation et l'intériorisation des signes véhiculés par les textes qui

3. Les textes signés Volochinov, comme ceux signés Medvedev, ont longtemps été attribués à Bakhtine sur la base des allégations de ce dernier. Les documents historiques aujourd'hui disponibles (cf. Bota \& Bronckart, 2008; Brandist, 2006, Bronckart \& Bota, 2011) montrent que Bakhtine a délibérément menti sur ce point et que son imposture a été "couverte" par ses promoteurs, pour des raisons financières et idéologiques. 
constitue la condition du développement psychologique permanent des personnes. Sous ce dernier angle, l'approche de Volochinov est d'une part compatible avec celle de Saussure, en ce que tous deux posent que les valeurs signifiantes de la textualité font l'objet d'une intériorisation, et se réorganisent donc nécessairement dans ce lieu d'ancrage que constitue la langue interne; elle pose d'autre part que les textes constituent un lieu de vie intermédiaire des représentations humaines, ou encore un lieu de mise en interface entre les représentations collectives et les représentations individuelles, telles que les avait analysées Durkheim.

\subsection{Des approches de l'architecture interne des textes}

Si elles n'avaient que partiellement été abordées par Volochinov (dans ses études des diverses formes de discours rapporté - op. cit., pp. 161220), les modalités de structuration interne des textes ont depuis lors fait l'objet de nombreuses études, ayant abouti à d'importants modèles de l'architecture textuelle, dont notamment celui élaboré par Adam dans une perspective plutôt ascendante (centrée sur l'articulation des propositions, des macro-propositions et des séquences) et celui élaboré par Rastier, dans une perspective clairement descendante (centrée sur les parcours isotopiques de significations). Nous nous permettrons d'évoquer ici notre approche propre (cf. Bronckart 1997b) qui est centrée sur les types de discours, comme entités structurantes intermédiaires, en ce qu'elles sont d'un côté infraordonnées eu égard aux genres et d'un autre côté constituent les cadres linguistiques de rang supérieur régissant la distribution d'une bonne part des marques syntaxiques et énonciatives. Ces types correspondent aux « modes d'énonciation » dont Genette (1986) avait clairement souligné qu'ils ne devaient pas être confondus avec les genres, et ils se construisent sur la base de deux types d'opérations. Les premières explicitent le rapport existant entre les coordonnées organisant le contenu thématique d'un texte et les coordonnées du monde externe dans lequel se déploie l'action langagière dont le texte est issu. Les secondes ont trait à la mise en rapport entre, d'une part les différentes instances d'agentivité (personnages, groupes, institutions, etc.) et leur inscription spatio-temporelle, telles qu'elles sont mobilisées dans un texte, et d'autre part les paramètres matériels de l'action langagière en cours (agent producteur, interlocuteur éventuel et espacetemps de production). Pour le premier type d'opération, soit les coordonnées 
du monde discursif sont présentées comme clairement disjointes de celles du monde de l'action langagière, soit cette mise à distance n'est pas opérée, et les deux sortes de coordonnées sont dès lors nécessairement conjointes. Cette première distinction revient ainsi à distinguer les mondes discursifs de l'ordre du RACONTER vs de l'ordre de l'EXPOSER. Pour le second type d'opération, soit un segment de texte explicite le rapport que ses instances d'agentivité entretiennent avec les paramètres matériels de l'action langagière, soit ce rapport n'est pas explicité et les instances d'agentivité entretiennent alors un rapport d'indifférence avec les paramètres de l'action langagière en cours. Une seconde distinction générale peut ainsi être posée entre les mondes discursifs exhibant, soit un rapport d'implication, soit un rapport d'autonomie, à l'égard des paramètres de l'action langagière. En croisant ces deux distinctions, on aboutit alors à l'identification de quatre mondes discursifs, qui sont traduits par ces configurations d'unités et de processus linguistiques que nous qualifions de types de discours: le monde de l'EXPOSER impliqué se réalise en discours interactif, le monde de l'EXPOSER autonome en discours théorique, le monde du RACONTER impliqué en récit interactif et le monde du RACONTER autonome en narration.

Notre modèle pose ensuite l'existence de divers mécanismes contribuant à l'instauration de la cohérence thématique (connexion, cohésion nominale) et de la cohérence interactive (voix et modalisations), procédés abondamment décrits en linguistique textuelle, mais que nous analysons dans leurs rapports de dépendance, soit avec les types de discours qu'ils "traversent", soit avec le genre dont relève l'ensemble du texte. Si ce modèle demeure bien évidemment imparfait et pourrait notamment, comme nous l'avons montré (cf. 2008), être complété par l'introduction de certains mécanismes mis en évidence par Rastier, pour la problématique qui nous occupe, l'important est que soient réellement pris en compte et analysés les niveaux de structuration intermédiaire de la textualité.

\section{Pour une linguistique au Service d'une sCience de L’humain}

Dès lors que l'on adhère à la thèse du rôle décisif que joue le langage dans la constitution et le développement de l'espace gnoséologique humain, la linguistique nous paraît pouvoir et devoir contribuer à la démonstration de trois des effets constitutifs de ce langage. 
Le premier concerne la constitution des unités de pensée, et l'analyse saussurienne des signes évoquée plus haut nous paraît en fait avoir déjà réglé la question. Dans la mesure où les signes sont arbitraires, ou n'ont aucun fondement substantiel, leur intériorisation aboutit à la constitution d'entités internes qui, à la différence des images du psychisme animal, ne sont plus dépendantes des conditions de renforcement du milieu; et cette autonomie leur confère une première caractéristique, de permanence et de stabilité (les représentations humaines persistent même lorsque s'éteignent les renforcements qui les ont suscitées). Ensuite, dès lors que la face signifiante du signe est constituée d'une image acoustique délimitée, le signifié qui y correspond se présente lui-même comme une entité mentale circonscrite; le signifié est, comme le soulignait De Mauro (1975, p. 438, note 128), un « analyseur » ou un " organisateur » qui fédère en une unité stable un ensemble d'images référentielles à caractère jusque-là idiosyncrasique. Et l'existence de telles unités constitue la condition sine qua non du déploiement des opérations de pensée (ces dernières requièrent en effet l'existence de termes stables auxquels s'appliquer). Enfin, les signes sont des entités dédoublées: ils sont constitués d'« enveloppes sociales » (selon la formule de Sapir, $1921 / 1953$, p. 20) qui renvoient à des ensembles d'images individuelles en même temps qu'elles les rassemblent, enveloppes dont la face sonore est par ailleurs perceptible et traitable; et c'est cette accessibilité d'entités à pouvoir dédoublant qui rend possible le retour de la pensée sur elle-même, ou encore la capacité de conscience. Ce à quoi il convient d'ajouter qu'étant donné l'origine sociale-conventionnelle des signifiants, la subsomption que ces derniers opèrent sur les images mentales idiosyncrasiques des individus ne peut jamais être complète, ou encore que ce processus laisse nécessairement du " reste représentatif » qui n'est évidemment pas sans rapport avec ce que l'on qualifie d'inconscient.

Pour aborder les deux autres types d'effets potentiels, qui restent quasi totalement à élucider, il nous paraît nécessaire de prendre appui d'abord sur des propositions ou des acquis émanant de trois auteurs déjà abondamment convoqués.

Saussure, s'il a surtout analysé des entités de la taille du mot, soutenait cependant que toutes les entités langagières relevaient de la sémiologie ( Sémiologie $=$ morphologie, grammaire, syntaxe, synonymie, rhétorique, stylistique, lexicologie, etc., le tout étant inséparable »-2002, p. 45). Ce qui signifiait pour lui que les syntagmes, les structures prédicatives ou les 
mécanismes de textualisation devaient exhiber des propriétés analogues à celles des signes-mots, dont notamment l'arbitraire radical, et donc témoigner d'une réelle autonomie eu égard à ces "correspondants" référentiels que constituent notamment les opérations cognitives. Cette hypothèse est peut-être trop forte, mais en tout état de cause, la question du taux d'arbitraire dont témoignent les entités supra-ordonnées aux mots mérite un sérieux examen, et est liée à la problématique des deux autres types d'effets potentiels qui seront évoqués ci-dessous.

Piaget a proposé une analyse des conditions de transformation de l'intelligence sensori-motrice en une pensée opératoire consciente que nous avons contestée, mais nous ne pouvons contester par contre son analyse des résultats de cette transformation, à savoir la construction d'opérations de pensée à caractère formel, organisées en une logique abstraite. Si, comme nous le soutiendrons ci-dessous, les opérations cognitives initiales (de 2 à 5 ans environ) procèdent directement de l'intériorisation des structures prédicatives de la langue d'usage et sont donc "marquées" par les propriétés particulières de cette langue, la mise en œuvre des processus d'abstraction réfléchissante et généralisation, telle que l'auteur l'a analysée, fait en sorte que se construisent progressivement ensuite des opérations dégagées des contraintes sémantico-syntaxiques du langagier, ou encore des opérations proprement cognitives, qui co-existent dès lors avec les opérations soustendant la gestion d'une langue particulière.

Vygotski a thématisé à sa manière cette co-existence, en soutenant que si le langage constitue le produit de la « fusion » de capacités communicatives et cognitives antérieures et "primaires », cette fusion n'est jamais totale, et que l'humain dispose donc nécessairement et d'une "pensée verbale » et d'une "pensée non verbalisée ». Et, ayant analysé, dans des corpus de textes, les décalages qui se produisent entre le plan des structures de signifiants formels et celui des construits cognitifs référés, il a soutenu que la perception et le traitement de ces décalages constituaient les conditions mêmes de la poursuite du développement psychologique:

\footnotetext{
«Partout — dans la phonétique, la morphologie, le lexique, la sémantique, et même dans la rythmique, la métrique et la musique — des catégories psychologiques se cachent derrière les catégories grammaticales ou formelles. Si dans un cas elles se recouvrent apparemment, dans d'autres elles divergent [...] Cette discordance, loin d'empêcher la pensée de se réaliser dans le mot, est la condition nécessaire pour que le mouvement de la pensée au mot soit possible. » (1934/1997, pp. 433-434)
} 
C'est sur ces bases que nous paraissent pouvoir être abordés les deux autres types d'effets constitutifs.

Comme Piaget l'avait relevé, les opérations cognitives de base relèvent de l'implication de significations: elles ont un caractère probabiliste ou normatif et ne peuvent pour cette raison dériver directement de la logique de nécessité des enchaînements causaux. Or ce caractère normatif se rencontre dans les structures prédicatives de base des langues naturelles, et plus précisément dans les modalités de réfraction que proposent ces structures du caractère probabiliste des actions humaines. Et deux questions méritent dès lors un sérieux examen empirique: par quels processus, dans un premier temps développemental, l'appropriation et l'intériorisation des structures prédicatives d'une langue génèrent-elles ces formes d'opérations imparfaites, ou déterminées linguistiquement et contextuellement, que Piaget a qualifiées d' " opérations concrètes »? Par quels processus, dans un second temps développemental, ces opérations peuvent-elles se dégager de ces déterminations pour devenir « formelles »?

S'agissant des opérations cognitives plus complexes, le même type de question peut être posé, en prenant en compte cette fois les niveaux de structuration intermédiaire de la textualité. De nombreux auteurs ont soutenu avec pertinence que le développement des identités personnelle ou sociale ne pouvait pas être sans rapport avec celui de la maitrise des mécanismes de gestion des voix et des modalisations requise par la production textuelle. Nous soutenons pour notre part que le développement de la maitrise des types de discours ne peut pas être sans rapport avec la construction des types de raisonnements (raisonnements par schématisation impliqués dans les discours interactifs; raisonnements causaux-chronologiques impliqués dans les récits et les narrations; raisonnements d'ordre logique et/ou semi-logique impliqués dans les discours théoriques), et nous soutenons également que la maîtrise des modalités de structuration temporelle propres aux types de discours constitue sans doute la condition du développement des diverses formes sociales d'appréhension et d'organisation du facteur Temps. Quelques recherches sont actuellement en cours sur ces thèmes (cf. Bulea \& Bronckart, 2006), mais le chantier est vaste ... 


\section{Conclusions}

Une science humaine adossée à l'épistémologie interactionniste sociale a besoin de la linguistique, comme nous espérons l'avoir montré. Mais pour conclure avec l'engagement qui a caractérisé cette intervention (et qui pourra peut-être, j'en conviens, paraitre présomptueux), nous nous permettrons d'énoncer trois convictions, ou trois conditions d'efficacité de la contribution souhaitée.

Accepter d'abord que tous les objets d'une science du langage ont une dimension fondamentalement sémiotique, que le langage et les langues sont avant tout des instruments de création de signification; " mettre la question du sens sous le paillasson » a évidemment constitué de ce point de vue une erreur fondamentale.

Accepter aussi que la réalité première du langage est d'ordre praxéologique; que le premier niveau empirique de la vie d'une langue est constitué des textes, dans leurs rapports aux activités humaines et à leur contexte, et que toute méthodologie d'analyse doit présenter dès lors un caractère descendant, des structures globales vers les structures et entités locales.

Reconsidérer enfin, dans une perspective réellement dialectique, les rapports entre les pratiques textuelles empiriques et ces vécus du langagier que constituent la langue personnelle et la langue de la communauté.

Recebido em junho de 2011 Aprovado em agosto de 2011 E-mail: jean-paul.bronckart@unige.ch

\section{BiBLIOGRAPHIE}

Adam, J.-M. (1990). Eléments de linguistique textuelle. Liège: Mardaga. Benveniste, E. (1974). L'appareil formel de l'énonciation. In Problèmes de linguistique générale, t. II (pp. 79-88). Paris: Gallimard.

Bloomfield, L. (1970). Le langage. Paris: Payot [Edition originale: 1933]. Bota, C. \& Bronckart, J.-P. (2008). Voloshinov et Bakhtine: deux approches radicalement opposées des genres de textes et de leur statut. Linx, 56, 67-73. 
BRANDIST, C. (2006). Early soviet research projects and the development of "bakhtinian" ideas: the view from the archives. Proceedings of the XII International Bakhtin Conference. University of Jyväskylä.

BRONCKART, J.-P. (1997a). Semiotic interaction and cognitive construction. Archives de Psychologie, 65, 95-106.

Bronckart, J.-P. (1997b). Activité langagière, textes et discours. Pour un interactionisme socio-discursif. Paris : Delachaux et Niestlé.

BroncKart, J.-P. (2008). Genres de textes, types de discours et "degrés" de langue. Hommage à François Rastier. Texto [http://www.revue-texto. net.], XIII, 1.

Bronckart, J.-P. \& Bota, C. (2011). Bakhtine démasqué. Histoire d'un menteur, d'une escroquerie et d'un délire collectif. Genève: Droz.

Bulea, E. \& Bronckart, J.-P. (2006). La saisie des compétences dans l'interprétation de l'activité de travail. Bulletin Vals/Asla, 84 [L. Mondada \& S. Pekarek Doehler (Ed.), La notion de compétence: études critiques], pp. 143-171.

Chomsky, N. (1969). Structures syntaxiques. Paris : Seuil [Edition originale: $1957]$.

Constantin, E. (2005). Linguistique générale. Cours de M. le professeur F. de Saussure. Cabiers Ferdinand de Saussure, 58, 71-289.

De Mauro, T. (1975). Notes. In Cours de linguistique générale (pp. 405-477). Paris: Payot.

Dilthey, W. (1992). Introduction aux sciences de l'esprit. In. Dilthey Euvres 1 (pp. 141-361). Paris : Editions du Cerf, [Edition originale: 1883].

Durkheim, E. (1898). Représentations individuelles et représentations collectives. Revue de métaphysique et de morale, 6, 273-302.

Genette, G. (1986). Introduction à l'architexte. In G. Genette et al., Théorie des genres (pp. 89-159). Paris : Seuil.

Habermas, J. (1987). Théorie de l'agir communicationnel, Tomes 1 et 2. Paris: Fayard.

Hauser, M.D., Chomsky, N. \& Tecumseh Fitch, W. (2002). The Faculty of Language: What Is It, Who Has It, and How did It Evolve. Science, 298, 1569-1579.

Kant, E. (1944). Critique de la raison pure. Paris : P.U.F. [Edition originale: $1781]$.

Komatsu, E. \& Wolf, G. (1996). Premier cours de linguistique générale (1907) d'après les cabiers d'Albert Riedlinger. Oxford/Tokyo: Pergamon. 
Piaget, J. (1970). Epistémologie des sciences de l'homme. Paris: Gallimard.

Piaget, J. (1989). L'explication en psychologie et le parallélisme psychophysiologique. In P. Fraisse \& J. Piaget (Ed.), Traité de psychologie expérimentale, Vol. I (pp. 137-184). Paris: PUF.

Rastier, F. (1989). Sens et textualité. Paris: Hachette.

Rastier, F. (2001). Arts et sciences du texte. Paris: PUF.

SAPIR, E. (1953). Le langage. Paris : Payot [Edition originale: 1921].

Saussure, F. (de) (1916). Cours de linguistique générale. Paris: Payot.

Saussure, F. (de) (2002). Ecrits de linguistique générale. Paris: Gallimard.

Spinoza, B. (de) (1954). L'Ethique. In Spinoza, Oeuvres complètes (pp. 301-596). Paris : Gallimard, [Edition originale: 1677].

Volochinov, V.N. (2010). Marxisme et philosophie du langage. Limoges: Lambert-Lucas [Edition originale: 1929].

Vygotski, L.S. (1997). Pensée et langage. Paris : La Dispute [Edition originale: $1934\}$. 